\title{
Review of: "Mapping native and non-native vegetation in the Brazilian Cerrado using freely available satellite products"
}

\author{
Yingying Dong ${ }^{1}$ \\ 1 Aerospace Information Research Institute (AIR)
}

Potential competing interests: The author(s) declared that no potential competing interests exist.

Native and non-native vegetation is very important for carbon analysis, especially in agriculture. Regional mapping products based on this paper ideas would be more helpful. 